\title{
Agent Strategy Generation by Rule Induction in Predator-Prey Problem
}

\author{
Bartłomiej Śnieżyński \\ AGH University of Science and Technology, \\ Department of Computer Science Kraków, Poland \\ Bartlomiej.Sniezynski@agh.edu.pl
}

\begin{abstract}
This paper contains a proposal of application of rule induction for generating agent strategy. This method of learning is tested on a predator-prey domain, in which predator agents learn how to capture preys. We assume that proposed learning mechanism will be beneficial in all domains, in which agents can determine direct results of their actions. Experimental results show that the learning process is fast. Multi-agent communication aspect is also taken into account. We can show that in specific conditions transferring learned rules gives profits to the learning agents.
\end{abstract}

Keywords: multi-agent systems, rule induction, machine learning.

\section{Introduction}

Decentralized problem solving becomes more and more popular. Architecture, which can be used for this purpose is multi-agent system. The problem is that in complex environments it is very difficult (or sometimes impossible) to specify and implement all system details a priori. A solution is a learning method application, which adopts the system to the environment. Learning can be also useful if the environment is not stationary.

To apply learning in a multi-agent system, one should chose a method of learning. There are many algorithms developed so far. However; in multi-agent systems most applications use reinforcement learning.

In this paper we show that rule induction can be successfully applied to generate agent strategy in the predator-prey domain, in which reinforcement learning and evolutionary computation methods were applied.

Rule induction is a supervised learning method, therefore, in the contrast to the methods mentioned above, it needs labeled examples (e.g. percepts-action pairs) to generate knowledge. We show, that in this domain an agent is able to generate such examples. The reason is that results of actions performed by learning agents are visible immediately. Any supervised learning method can be applied in a similar way in all environments with such a property.

In the following sections, related research considering learning in multi-agent systems is briefly discussed, the developed system is described, and experimental results are presented and analyzed. 


\section{Learning in Multi-agent Systems}

Good survey of learning in multi-agent systems can be found in 1 and 2 . As it was mentioned above, the most popular learning technique used is reinforcement learning that allows to learn agent strategy: what action should be executed in a given situation. Other techniques can be also applied: neural networks, models coming from game theory as well as optimization techniques (like the evolutionary approach, tabu search etc.). However, optimization techniques improve performance of the system using many populations of agents instead of a single agent.

Reinforcement learning allows to generate a strategy for an agent in a domain, in which the environment provides some feedback after the agent has acted. Feedback takes the form of a real number representing reward, which depends on the quality of the action executed by the agent in a given situation. The goal of the learning is to maximize estimated reward. This method was successfully applied in the predator-prey domain [3. In this work predator agents use reinforcement learning to learn a strategy minimizing time to catch a prey. Additionally, agents can cooperate by exchanging sensor data, strategies, or episodes. Experimental results show that cooperation is beneficial. Other researchers working on this domain successfully apply genetic programming 4] and evolutionary computation [5].

There is only a few works known to the author on supervised learning in multi-agent systems. Rule induction is used in a multi-agent solution for vehicle routing problem [6]. However; in this work learning is done off-line. First, rules are generated by $\mathrm{AQ}$ algorithm (the same as used in this work) from traffic data. Next, agents use these rules to predict traffic. In [7, agents learn coordination rules, which are used in coordination planning. If there is not enough information during learning, agents can communicate additional data during learning. Airiau [8 adds learning capabilities into BDI model. Decision tree learning is used to support plan applicability testing.

In 9 there is a comparison of supervised learning and reinforcement learning methods in a Fish-Banks game. Agents run fishing companies and learn how to allocate ships. In this case, agents using rule induction perform slightly better than ones using reinforcement learning.

Universal architecture for learning agent can be found in 10. It fits mainly reinforcement learning. Sardinha et. al, propose a learning agent design pattern, which can be used during system implementation [11. More abstract architecture, which is used in this work, is presented in [12].

\section{System Architecture}

\subsection{Predator-Prey Domain}

Predator-prey domain is a simple simulation with two types of agents: predators, and preys. The aim of a predator is to hunt for a prey. Environment is a grid world with size $n \times n$ with "glued" opposite edges (it is a torus). Time is discrete, 
its flow is represented by turns. In every turn all agents receive percept data from the environment and chose their actions, which are next executed.

Agents can move in the four directions (up, down, left, and right) or not move at all. If predator occupies a field next to the prey, the prey is captured.

Predators have limited range of sight. If prey is closer than a given threshold, predator gets information about type of the prey, and its relative position. If more then one prey is in range, only the closer one is visible.

Two types of the preys are defined: bird and mouse. First one moves up, down or does not move at all with equal probability. Similarly, mouse move left, or right, or does not move.

Two types of predators are defined: random and learning. The former moves in the four directions or do not move with equal probability. The latter uses rule induction to improve performance.

\subsection{Learning Predator}

Learning predator architecture is presented in Fig. 1, In this application percepts received by the agent is nul if no prey is in the observation range, or is a triple $(t, d x, d y)$, where $t$ is a type of a prey, and $d x, d y$ are relative coordinates of the prey along $\mathrm{X}$ and $\mathrm{Y}$ axis, respectively.

Initially, processing module selects actions from the set \{none, up, down, left, right\} randomly. If, after actions execution, distance to the prey decreases, new example is stored in the training data memory. Percept triple from the previous round form attribute values of the example and action executed is its class. Here important property of the environment is used. It is possible to generate training data because action results are immediately visible.

Rules generated during learning have a form $p_{1}, p_{2}, \ldots, p_{n} \rightarrow a$, where $p_{i}$ are tests on the attributes representing current percepts, and $a$ is an action, which should be executed.

During a learning phase training data is sent to the learning module. In this research AQ21 rule induction program is used [13. It is the latest implementation

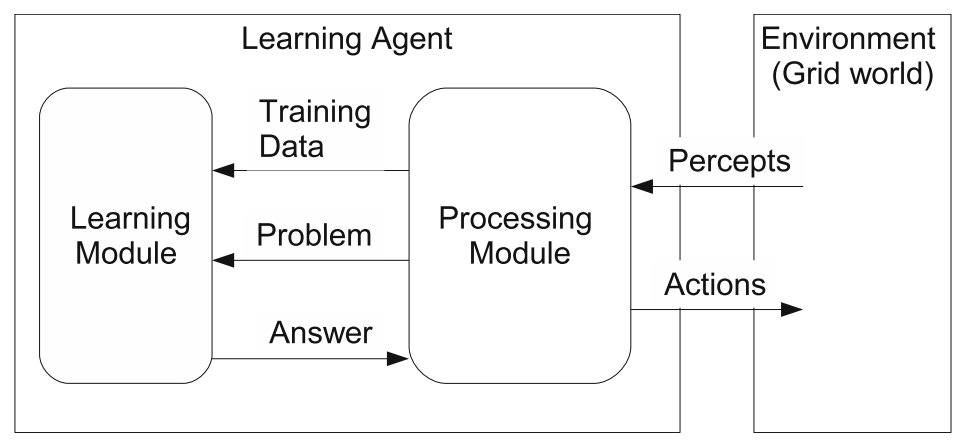

Fig. 1. Learning agent architecture 
of AQ algorithm [14]. Rules are generated using sequential covering: the best rule (e.g. giving correct answer for the most examples) is constructed by a beam search, examples covered by this rule are eliminated from the training set, and the procedure repeats.

AQ21 program is executed in a theory formation mode. It means that rules generated are sound and complete. Ambiguity option is set to IncludeInMajority. If there are more events in the training data with the same values of all attributes and different classes (actions), learning algorithm assigns to the event the majority class. All other options are set to their default values.

In the future various option settings can be tested. However; it is one of advantages of rule induction application for agent strategy generation: tuning of parameters is not necessary to get good results.

If knowledge is generated, processing module can use learning module to chose an action in the current situation. Input to the learning module is a problem, which consists of a percept triple, and output - answer is a conclusion of the rule matching the percepts or nul if the rule is not found (in such a case agent executes random action).

In this research two types of communication were considered for the predator agent: exchange of generated rules and exchange of the training data. If no matching rule is found, predator agent can ask another agent for a matching rule and store it in its knowledge base. Exchange of the training data means that during learning agent asks for examples stored in other agent's memory to generate better knowledge. During experiments only the former case was tested. Results are initial, but we can show that in specific conditions transferring learned rules gives profits to the learning agents.

\subsection{Implementation}

The software used in experiments is written in Prolog, using Prologix compiler [15]. Prologix is an extension of BinProlog that has many powerful knowledge-based extensions (e.g. agent language LOT, Conceptual Graphs and KIF support). AQ21 program [13] is executed from Prolog and rules generated are added to the code as additional horn clauses.

\section{Experimental Results}

\subsection{Setup}

Let us introduce several definitions. Game is defined as a sequence of turns beginning with the initial positions of agents and ending in a turn when all preys are captured. Twelve consecutive games is called a sequence. It is assumed that memory and knowledge base of learning predators is kept unchanged between games in a sequence. However, it is cleared between sequences. Performance of predators is measured by a number of turns in a game; the less, the better. 


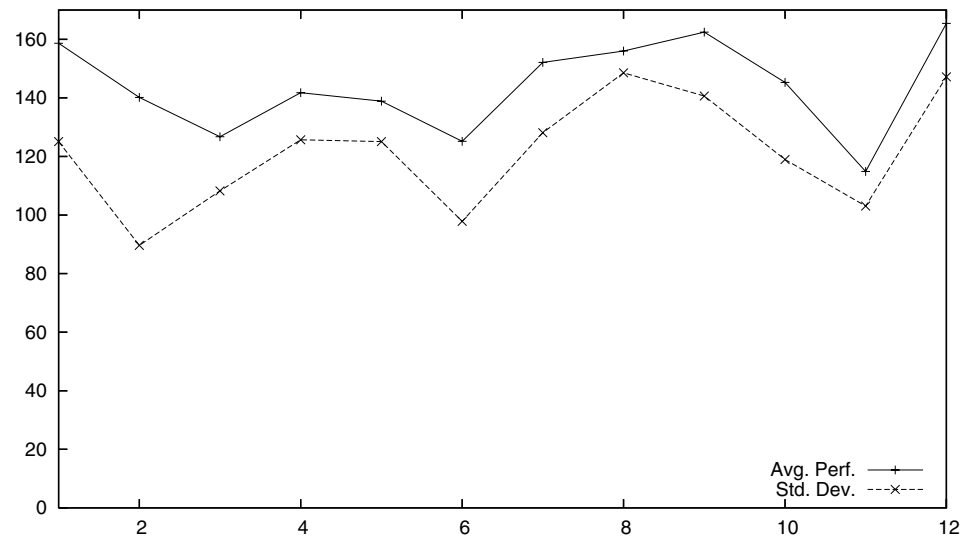

Fig. 2. Average performance and its standard deviation for random agents in consecutive games

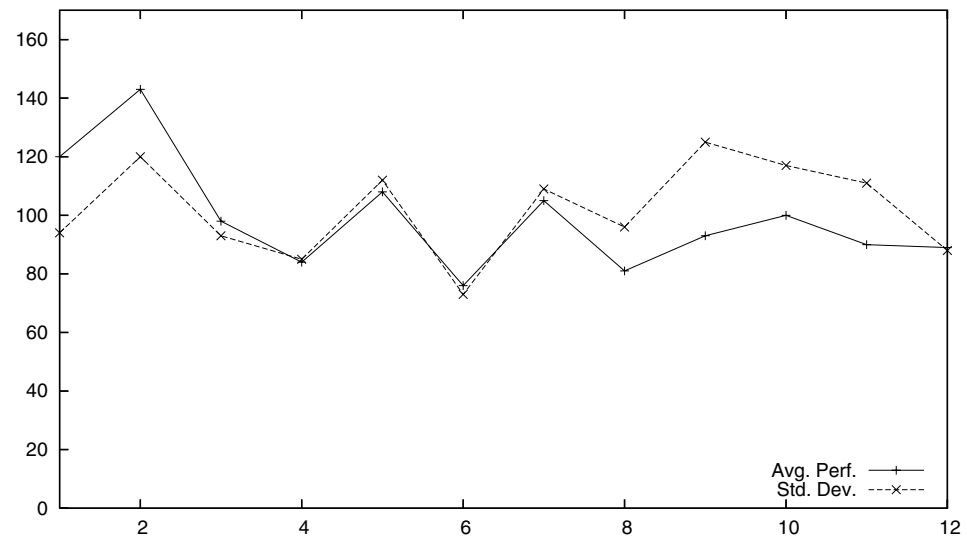

Fig. 3. Average performance and its standard deviation for learning agents (without communication) in consecutive games

Grid world in experiments has dimensions $16 \times 16$. Initial positions of predators are $(0,0)$ and $(8,8)$ to maximize distance between them. There are two prey agents: bird and mouse. In all experiments 60 sequences of games are executed and performance measures in every game are stored. Learning predators execute learning algorithm at the end of every even game.

\subsection{Random Predators}

The aim of the first experiment is to test performance of random predators. Two of them take part in the games. Initial positions of preys are random. The average performance of the predators is 140.01. Its standard deviation is 
very high: 120.50. Hence, as we can see in Fig. 2, average performance varies from game to game in the sequence.

\subsection{Learning Predators}

Two learning predators take part in this experiment. They do not communicate. Initial positions of preys are random. Average performance measures in consecutive games are presented in Fig. 3 .

The learning process is fast. In this experiment after executing about 300 random actions in two games, and collecting examples, performance changes from about 130 to 90 .

As we can see, performance of learning agents increases rapidly at the beginning of the learning process, when generated rules are used instead of a

(a)

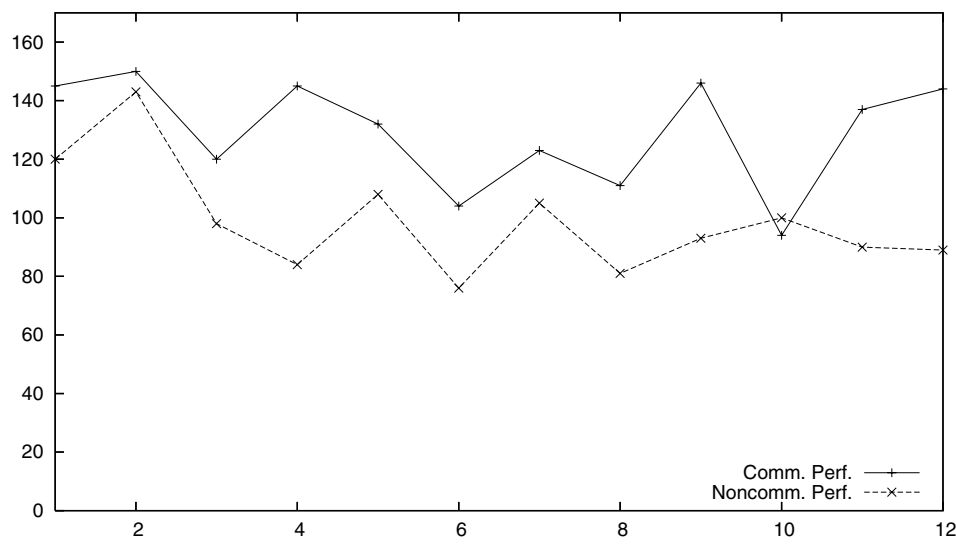

(b)

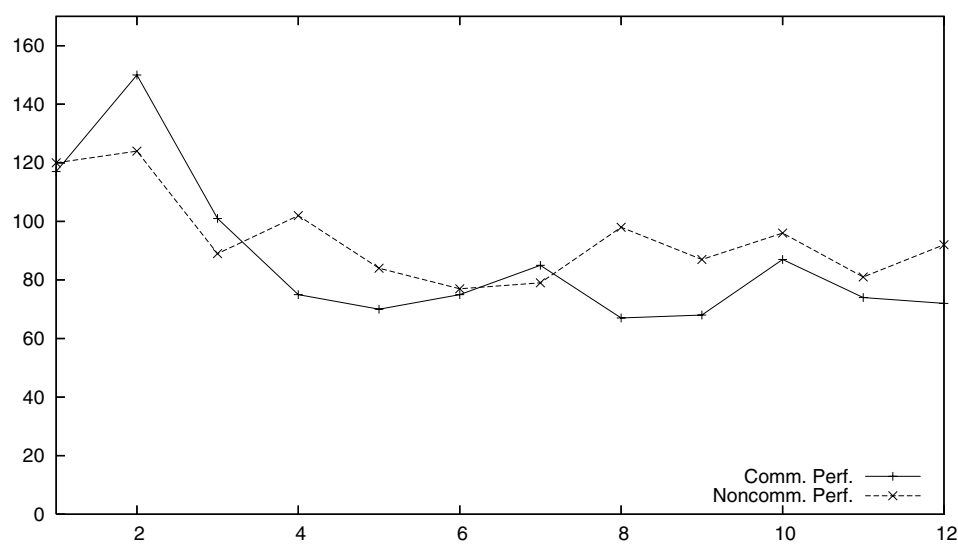

Fig. 4. Performance comparison or learning agents with and without communication in experiment with random initial prey positions (a) and fixed initial prey positions (b) 
random choice. Next it increases slowly, because new examples do not contain any significant knowledge. The performance stabilizes at the end of the process.

\subsection{Learning Predators with Communication}

This experiment tests influence of communication. In Fig.44(a) we can see comparison of performances of communicating and non-communicating predators with random initial positions of preys. As we can see, communication not only does not help learning agents, but even makes the performance worse. Unfortunately, the reason of this behavior is not clear yet.

We tested another initial setting. In these experiments bird-prey starts in position $(3,0)$ with probability 0.8 and in $(11,8)$ with probability 0.2 , and mouse-prey vice versa. In Fig. 4 (b) we can see performance comparison for this settings. Performance of communicating agents is better than ones without communication.

This result suggests that communication is profitable if a learning agent is rarely in the situation, which is common for the other agent. In such conditions the other agent can provide appropriate, useful knowledge to transmit and use.

\subsection{Rule examples}

Examples of rules learned by the predator-agent in a form of Prolog clauses are presented in Fig. 5. They can be interpreted in the following way. Both rules have action left in the conclusion. $\mathrm{S}$ is an identifier of a state, for which decision should be made. Rule (a) is applicable if the predator sees a bird which has the same or smaller by one $x$ coordinate and relative vertical position is between -3 and 0 . Premise of rule (b) checks if the predator sees a mouse, relative $x$ coordinate is -2 , and $y$ is between -3 and -2 .

(a)

$\operatorname{dir}(\mathrm{S}$, left $):-$
type $(\mathrm{S}$, bird $)$,
$\operatorname{dx}(\mathrm{S}, \mathrm{X})$,
$\mathrm{X}>=-1$,
$\mathrm{X}=<0$,
$\operatorname{dy}(\mathrm{S}, \mathrm{Y})$,
$\mathrm{Y}>=-3$,
$\mathrm{Y}=<0$.

(b)

$$
\begin{aligned}
& \operatorname{dir}(\mathrm{S}, \text { left }):- \\
& \text { type }(\mathrm{S}, \text { mouse }), \\
& \operatorname{dx}(\mathrm{S},-2), \\
& \operatorname{dy}(\mathrm{S}, \mathrm{Y}), \\
& \mathrm{Y}>=-3, \\
& \mathrm{Y}=<-2 .
\end{aligned}
$$

Fig. 5. Examples of rules (in the form of Prolog clauses) learned by the agent

\section{Conclusion and Further Research}

In this paper idea of the agents using rule induction for learning strategy is presented. Solution proposed is tested on a specific example - predator-prey domain. 
However, similar strategy generation works also for a Fish-Banks game [16]. The only condition for the environment to use this method is that agent should be able to observe direct results of its actions. In other words, results are not delayed and there is no reward assignment problem. We assume that rule induction, and more general, supervised learning can be applied in such circumstances in other domains.

Results for the Fish-Banks game [9] and initial results for the predator-prey problem [17] show that supervised learning give improvements faster than reinforcement learning.

Advantage of rule induction is clarity of rule-based knowledge representation. It is possible to interpret the knowledge base or check its correctness manually. It can be very important for some domains.

Additionally, because rule-based knowledge representation is modular, exchange of the knowledge is easy and has low cost. Only necessary rules can be transmitted.

A problem discovered in this research is a high value of standard deviation of performance measure in predator-prey domain. It makes this domain not very attractive for comparison of various methods. In the future research this domain should be modified and/or other domains will be explored.

Interesting issue for further research is developing agents using several learning methods for different aspects of their activity. Also exchange of training data as an another form of communication during learning should be tested. And last but not least, performance of rule learning agent should be compared to the performance of reinforcement learning agent in the same conditions.

Acknowledgments. The author is grateful to Arun Majumdar, Vivomind Intelligence Inc. for providing Prologix system (used for implementation), and for help with using it, Janusz Wojtusiak, MLI Laboratory, George Mason University for AQ21 software and assistance, and last but not least Jens Pfau, Technische Universität Darmstadt for suggesting predator-prey domain, and implementation of the first, Java version of the system using decision-tree induction and reinforcement learning.

\section{References}

1. Stone, P., Veloso, M.: Multiagent systems: A survey from a machine learning perspective. Autonomous Robots 8, 345-383 (2000)

2. Panait, L., Luke, S.: Cooperative multi-agent learning: The state of the art. Autonomous Agents and Multi-Agent Systems 11 (2005)

3. Tan, M.: Multi-agent reinforcement learning: Independent vs. cooperative agents. In: Proceedings of the Tenth International Conference on Machine Learning, pp. 330-337. Morgan Kaufmann, San Francisco (1993)

4. Haynes, T., Sen, I.: Evolving behavioral strategies in predators and prey. In: Adaptation and Learning in Multiagent Systems, pp. 113-126. Springer, Heidelberg (1996)

5. Giles, C.L., Jim, K.-C.: Learning communication for multi-agent systems. In: WRAC, pp. 377-392 (2002) 
6. Gehrke, J.D., Wojtusiak, J.: Traffic prediction for agent route planning. In: Bubak, M., van Albada, G.D., Dongarra, J., Sloot, P.M.A. (eds.) ICCS 2008, Part III. LNCS, vol. 5103, pp. 692-701. Springer, Heidelberg (2008)

7. Sugawara, T., Lesser, V.: On-line learning of coordination plans. In: Proceedings of the 12th International Workshop on Distributed Artificial Intelligence, pp. 335345, 371-377 (1993)

8. Airiau, S., Padham, L., Sardina, S., Sen, S.: Incorporating learning in bdi agents. In: Proceedings of the ALAMAS+ALAg Workshop (May 2008)

9. Śnieżyński, B.: Resource management in a multi-agent system by means of reinforcement learning and supervised rule learning. In: Shi, Y., van Albada, G.D., Dongarra, J., Sloot, P.M.A. (eds.) ICCS 2007. LNCS, vol. 4488, pp. 864-871. Springer, Heidelberg (2007)

10. Russell, S., Norvig, P.: Artificial Intelligence - A Modern Approach. Prentice-Hall, Englewood Cliffs (1995)

11. Sardinha, J., Garcia, A., Milidi, R., Lucena, C.: The agent learning pattern. In: Fourth Latin American Conference on Pattern Languages of Programming, SugarLoafPLoP 2004, Brazil (2004)

12. Śnieżyński, B.: An architecture for learning agents. In: Bubak, M., van Albada, G.D., Dongarra, J., Sloot, P.M.A. (eds.) ICCS 2008, Part III. LNCS, vol. 5103, pp. 722-730. Springer, Heidelberg (2008)

13. Wojtusiak, J.: AQ21 User's Guide. Reports of the Machine Learning and Inference Laboratory, MLI 04-3. George Mason University, Fairfax, VA (2004)

14. Michalski, R.S., Larson, J.: Aqval/1 (aq7) user's guide and program description. Technical Report 731, Department of Computer Science, University of Illinois, Urbana (June 1975)

15. Majumdar, A., Tarau, P., Sowa, J.: Prologix: Users guide. Technical report, VivoMind LLC (2004)

16. Śnieżyński, B., Koźlak, J.: Learning in a multi-agent approach to a fish bank game. In: Pěchouček, M., Petta, P., Varga, L.Z. (eds.) CEEMAS 2005. LNCS, vol. 3690, pp. 568-571. Springer, Heidelberg (2005)

17. Pfau, J., Śnieżyński, B.: Comparison of reinforcement and supervised learning in the predator prey game (2008) (unpublished) 